\title{
Estudio Preliminar Sobre La Decoloración Del Residual Líquido De La Producción De Papel Mediante Ozonización
}

Ing. Agustín Leiva Pérez, Ph.D.

Docentes - Investigadores de la Escuela Superior

Politécnica Agropecuaria de Manabí - MFL.

Dra. Sonia E. Peñafiel Acosta, M.Sc.

Docentes - Investigadores de la Escuela Superior Politécnica de Chimborazo

Ing. Joffre A. Andrade Candell, M.Sc.

Docentes - Investigadores de la Escuela Superior

Politécnica Agropecuaria de Manabí - MFL.

Q. F. Ana M. Aveiga Ortiz,

M.Sc., Dr. Guido G. Brito Zuñiga, M.Sc

Docentes - Investigadores de la Escuela Superior

Politécnica de Chimborazo

Teódulo R. Zambrano Farías, M.Sc

Docentes - Investigadores de la Escuela Superior

Politécnica Agropecuaria de Manabí - MFL

Abstract

Preliminarily, the relationship between Color Reduction $\left(\% R_{C}\right)$, Total Suspended Solids concentration (SST), and as independent variable, the time of Ozone Oxidation Process, was evaluated with reference values of $D Q O$ of the black liquor adjusting its concentration from SST to 300 and $50 \mathrm{mg} / \mathrm{dm}^{3}$. A batch reactor of $12,60 \mathrm{dm}^{3}$, an effective height of $3,2 \mathrm{dm}$ and an ozone generating device from SEFILTRA company (air flow of $2 \mathrm{dm}^{3} / \mathrm{min}, \mathrm{c}\left(\mathrm{O}_{3}\right)$ of $9,50 \mathrm{mg} / \mathrm{dm}^{3}$ with a $\mathrm{O}_{3}(\mathrm{~g})$ production $\left.19 \mathrm{mg} / \mathrm{min}\right)$ was used. The polynomic correlation was determined from 192 data sets with satisfactory adjustment level $(\mathrm{R}=0,92 ; \mathrm{p}<0,001)$. Although, preliminarily, the $D Q O$ reduction $\left(\% R_{D Q O}\right)$ was not included in the polynomial relationship, it can be concluded that:

a) The $\% R_{C}$ and $\% R_{D Q O}$ is reduced when color and $D Q O$ increases from the ozonized sample at same time intervals; this reduction is greater when the SST levels of the ozonized sample increases. 
b) The $\% R_{C}$ and $\% R_{D Q O}$ decrease when the SST level of the ozonized sample increases.

c) Depending on the initial color, of the $D Q O$ and of the SST level of the sample, values from 10 to $53 \% R c$ were obtained after $10 \mathrm{~min}$, and of 66 to $94 \% R c$ after $60 \mathrm{~min}$.

Keywords: Color reduction, solid suspended concentration

\section{Resumen}

Se evaluó, preliminarmente, la relación entre la reducción del color $\left(\% R_{C}\right)$, la concentración de Sólidos Suspendidos Totales (SST), y, como principal variable independiente, el tiempo del proceso de ozonización, con valores de la Demanda Química de Oxígeno $(D Q O)$ referenciales, del licor negro, ajustando su concentración de SST a 300 y $50 \mathrm{mg} / \mathrm{dm}^{3}$. Se empleó un reactor batch de $12,60 \mathrm{dm}^{3}$, una altura efectiva de $3,2 \mathrm{dm}$ y un equipo generador de ozono de la firma española SEFILTRA (flujo de aire de 2 $\mathrm{dm}^{3} / \mathrm{min}$, c $\left(\mathrm{O}_{3}\right)$ de $9,50 \mathrm{mg} / \mathrm{dm}^{3}$ con una producción de $\mathrm{O}_{3}(\mathrm{~g})$ de $\left.19 \mathrm{mg} / \mathrm{min}\right)$. Se determinó el polinomio de correlación, partiendo de 192 juegos de datos con nivel de ajuste satisfactorio $(R=0,92 ; p<0,001)$. Aunque preliminarmente no se incluyó en la relación polinómica hallada al porcentaje de la reducción de DQO $\left(\% R_{D Q O}\right)$, se concluyó que:

a) Los $\% R_{C}$ y $\% R_{D Q O}$ disminuyen al aumentar el color y la $D Q O$ de la muestra a ozonizar en un mismo intervalo de tiempo; esta reducción es más marcada al ser mayor el nivel de SST de la muestra a ozonizar.

b) Los $\% R_{C}$ y $\% R_{D Q O}$ descienden al incrementarse el nivel de $S S T$ de la muestra a ozonizar.

c) Dependiendo del color inicial, de la $D Q O$ y del nivel de $S S T$ de la muestra, se obtuvieron valores del $\% R_{C}$ de 10 a $53 \%$ al cabo de 10 min y de 66 a $94 \%$ al transcurrir $60 \mathrm{~min}$.

Mots clés: Reducción del color, sólidos Suspendidos Totales

\section{Introducción}

Uno de los parámetros más importantes, al considerar la agresividad ambiental de un residual líquido, es su color debido a que en ocasiones presenta persistencia en las corrientes de aguas, aún a grandes distancias del punto de impacto o vertimiento. En la industria productora de papel y cartón $^{1}$, al separar la pulpa (parte insoluble) de la soluble, ésta consiste de una corriente residual de elevada coloración y Demanda Química de

${ }^{1}$ Chowdury, D. A.; Wikman, B. (2014) Colloidal Stability in Pulp and Paper Industry Process Waters. European Scientific Journal 
Oxígeno (DQO), debido a la presencia de ligninas, polímeros insolubles en ácidos y solubles en álcalis fuertes, como por ejemplo, $\mathrm{Na}(\mathrm{OH})$.

Debido a la poca bioxidabilidad de estos polifenoles, resulta atractivo el tratamiento de decoloración y, por lo tanto, de oxidación química, mediante la aplicación de un oxidante muy enérgico como lo es el ozono, $\mathrm{O}_{3}(\mathrm{~g})$. El objetivo principal de este trabajo fue la evaluación de la decoloración del residual líquido de la producción de papel de bagazo, conocido como licor negro, mediante ozonización.

\section{Fundamerntación teórica}

$\mathrm{El}$ ozono, $\mathrm{O}_{3}(\mathrm{~g})$ es un gas incoloro con un tono ligeramente azulado y tiene un característico olor acre que puede resultar irritante, formándose en la naturaleza como resultado de las descargas eléctricas producidas en las tormentas y en las capas altas de la atmósfera, particularmente en la troposfera, como consecuencia de la acción de los rayos ultravioleta sobre las moléculas del oxígeno biatómico, $\mathrm{O}_{2}(\mathrm{~g})$. El ozono se descompone fácilmente para formar oxígeno biatómico, en un proceso claramente exoenergético, de acuerdo con la siguiente ecuación química, que representa al proceso: ${ }^{2}$

$$
2 \mathrm{O}_{3} \rightarrow 3 \mathrm{O}_{2} \quad \Delta \mathrm{H}_{0}=-144 \mathrm{~kJ} / \mathrm{mol}
$$

Se produce también un incremento significativo de entropía, por lo que se explica por qué en la superficie terrestre, el ozono es altamente inestable. ${ }^{3}$ Se comporta como un oxidante enérgico, capaz de estabilizar compuestos tan complejos como la lignina (gran asociación de polifenoles).

$$
\mathrm{C}_{9} \mathrm{H}_{10} \mathrm{O}_{2}, \mathrm{C}_{10} \mathrm{H}_{12} \mathrm{O}_{3}, \mathrm{C}_{11} \mathrm{H}_{14} \mathrm{O}_{4}
$$

La lignina es un polímero de naturaleza aromática con alto peso molecular que tiene como base estructural unidades de fenil-propano y probablemente está ligada a los polisacáridos (poliosas) de la madera.

\section{Composición elemental de la lignina}

Es un hecho comprobado que en la composición química elemental de la lignina aparecen única y exclusivamente carbono, hidrógeno y oxígeno. La composición básica elemental en términos de porcentajes varía particularmente si la lignina es obtenida de árboles coníferos o latifoliados, o de bagazo de caña y, de cómo ha sido el método de separación de la misma.

\footnotetext{
${ }^{2}$ EPA (2007) Part III, 40 CFR, Part 122, 136 et al. Guidelines Establishing Test Procedures for the Analysis of Pollutants Under the Clean Water Act: National Primary Drinking Water regulations; and National Secondary Drinking Water Regulations; Analysis and Sampling Procedures; Final Rule.

${ }^{3}$ Wark, K; Warner, C.F. (1997). Contaminación del aire. Origen y control. Ed. Limusa. México.
} 


\section{Base Estructural de la Lignina}

La base estructural de la lignina es el fenil-propano, teniendo enlazado al anillo bencénico un número variable de grupos hidroxílicos y metoxílicos. En el anexo se muestra la estructura aproximada de la lignina.

\section{Objetivo}

Evaluar la relación entre la concentración de Sólidos Suspendidos Totales, la remoción de color y el tiempo de ozonización del licor negro residual de la industria productora de papel y cartón.

\section{Materiales y métodos Materiales}

Los materiales empleados durante esta investigación, además de los correspondientes al laboratorio, fueron los siguientes:

- $\quad$ Licor negro crudo de la producción de papel (separada la lignina con $\mathrm{NaOH}$, medio fuertemente básico).

- Reactor batch de plástico oscuro en forma cilíndrica circular recta, con volumen total de $12,60 \mathrm{dm}^{3}$ (aproximadamente $2 \mathrm{dm}$ de diámetro de base y $4 \mathrm{dm}$ de altura) con entrada y salida de gases, y toma de muestra en el punto central de su altura efectiva $(3,2 \mathrm{dm})$.

- $\quad$ Equipo Generador de Ozono SEFILTRA, Madrid, España, que utiliza aire como insumo de $\mathrm{O}_{2}$ y cuyas características principales de interés para el estudio se muestran en el Cuadro 1.

- $\quad$ Equipo de filtración común.

- $\quad$ Filtros para análisis gravimétrico: AP40 Millipore.

- $\quad$ Mangueras plásticas de $\mathrm{PVC}$ de $0,5 \mathrm{~cm}$ de diámetro interior.

\section{Métodos}

Para este propósito se implementó un reactor químico batch descrito anteriormente, estableciéndose dos niveles de concentración de SST (50 y $300 \mathrm{mg} / \mathrm{dm}^{3}$ ), mediante operaciones de filtración, cada uno con tres diferentes niveles de color (en los alrededores de 0,9822;0,7321;0,6258), correspondientes a concentraciones de DQO en el entorno de 23389, 11514, y $10040 \mathrm{mg} / \mathrm{dm}^{3}$. Fijados los tiempos de reacción (ozonización) en 10, 60, y 180 minutos, se extrajeron tres muestras de $10 \mathrm{~cm}^{3}$ para cada uno de los tres tiempos de operación fijados, calculándose las eficiencias de remoción (\%) de color y DQO.

Las mediciones de color fueron referidas a opacidad, o sea (1 Y/100), donde Y es la luminiscencia en el método del triple estímulo con 10 ordenadas $^{4,5}$. Este y todos los demás análisis químicos y físicos fueron

\footnotetext{
${ }^{4}$ CIE 15. (2004) Technical report, colorimetry. Commission Internationale de L’Eclairage.
} 
realizados siguiendo las técnicas establecidas por APHA, AWWA y WPCF $(2005)^{6}$.

Cuadro 1. Características principales utilizadas del equipo Generador de Ozono SEFILTRA,

\begin{tabular}{|c|c|c|c|}
\hline $\begin{array}{c}\text { FLUJO AIRE } \\
\left(\mathbf{d m}^{\mathbf{3}} / \mathbf{m i n}\right)\end{array}$ & $\begin{array}{c}\mathbf{c}\left(\mathbf{O}_{\mathbf{3}}\right) \\
\left(\mathbf{m g} / \mathbf{d m}^{\mathbf{3}}\right)\end{array}$ & $\begin{array}{c}\text { PRODUCCIÓN DE O } \\
\mathbf{3}\end{array}$ & $\boldsymbol{\%}$ PESO \\
\hline 2 & 9,50 & 19 & 0,8 \\
\hline
\end{tabular}

\section{Resultados y discusión}

En el Cuadro 2 se puede ver algunos de los resultados obtenidos. Se determinó que el nivel de SST del licor negro sedimentado generaba un efecto negativo en el proceso y, se observó en aguas residuales similares, en cuanto a color y $D Q O$, un incremento de los porcentajes de remoción de dichos parámetros, al disminuir de 300 a $50 \mathrm{mg} / \mathrm{dm}^{3}$ el nivel de los $S S T$ de la muestra a ozonizar, para un mismo tiempo de reacción y disposición similar de ozono.

También se encontró que para un mismo nivel de muestra e iguales tiempos de reacción, un incremento de la eficiencia de eliminación de color y de $D Q O$, a medida que se hace menos agresivo el residual líquido bajo estudio.

Todo lo planteado anteriormente se debe, principalmente, a que este es un proceso controlado por la difusión del gas en el seno del líquido ${ }^{7}$, es decir, que las reacciones químicas ocurren en una de las fases, en este caso la líquida, después que se ha efectuado la transferencia de ozono de la fase gaseosa a la líquida, lo cual conduce a:

- bajos valores de la energía de activación,

- $\quad$ efecto poco apreciable de la temperatura sobre la velocidad del proceso y,

- $\quad$ significativo efecto de la turbulencia sobre la velocidad del proceso.

${ }^{5}$ Hunter Lab. (2001) Principios básicos de medida y percepción de color. Información técnica. HUNTER LAB.

6 APHA, AWWA-WPCF. (2005) Standard Methods for the Examination of Water and Wastewater. 21th Edition. New York.

${ }^{7}$ Levenspiel, O. (1971) Ingeniería de las Reacciones Químicas. Ed. Reverté, Barcelona, España. 
Cuadro 2. Resultados preliminares condensados sobre la variación de la eficiencia de remoción de color y $D Q O$ con las características iniciales del licor negro sedimentado, para diferentes tiempos de ozonización (10, 60 y $180 \mathrm{~min}$ ).

\begin{tabular}{|c|c|c|c|c|c|c|c|c|}
\hline SST & $\mathrm{C}_{0}$ & DQOo & $\% \mathrm{R}_{\mathrm{C} 10}$ & $\% \mathrm{R}_{\mathrm{DQO10}}$ & $\% \mathrm{R}_{\mathrm{C} 60}$ & $\% \mathrm{RDQO60}$ & $\% \mathrm{R}_{\mathrm{C} 180}$ & $\% R_{D Q O 180}$ \\
\hline 300 & 0,9822 & 23389 & 8,06 & 3,01 & 60,02 & 44,30 & 83,40 & 72,22 \\
\hline 300 & 0,7321 & 11514 & 18,64 & 14,29 & 71,30 & 68,73 & 93,04 & 78,01 \\
\hline 300 & 0,6258 & 10040 & 43,90 & 23,44 & 78,44 & 71,12 & 93,94 & 78,96 \\
\hline 50 & 0,9910 & 26322 & 24,18 & 13,16 & 80,00 & 63,13 & 98,03 & 91,69 \\
\hline 50 & 0,8183 & 12226 & 39,02 & 33,14 & 85,80 & 78,25 & ------ & ------ \\
\hline 50 & 0,6436 & 10302 & 63,10 & 46,20 & 94,82 & 89,92 & 98,59 & 93,19 \\
\hline
\end{tabular}

$S S T=$ Sólidos Suspendidos Totales $\left(\mathrm{mg} / \mathrm{dm}^{3}\right)$

$C_{0}=$ Color inicial del licor negro sedimentado, como opacidad, o sea $(1-\mathrm{Y} / 100)$, donde $\mathrm{Y}$ es la luminiscencia en el método del triple estímulo con 10 ordenadas.

$D Q O_{0}=$ Demanda Química de Oxígeno inicial del licor negro filtrado $\left(\mathrm{mg} / \mathrm{dm}^{3}\right)$.

$\% R c=$ Porcentaje de Reducción de Color

$\% R_{D Q O}=$ Porcentaje de Reducción de DQO

Debido a ello, se espera un aumento de la efectividad de la operación, al incrementar el gradiente de velocidad a valores tales que produzcan un Número de Reynolds modificado para agitación de 10000 a 20000, según las características del reactor usado, de manera que se alcance el punto de transición hacia condiciones de cinética química limitante.

Por último se presenta la correlación hallada según 192 juegos de datos que involucran las eficiencias de eliminación de color y $D Q O$, el color inicial y, la concentración de SST del licor negro sedimentado, así como el tiempo de ozonización.

El polinomio de correlación hallado fue:

$$
\begin{aligned}
Y=173,90 & -254,07 X_{1}-0,59 X_{2}+1,24 X_{3}+120,07 X_{1}^{2}+0,01 X_{1} X_{2} \\
& +0,15 X_{1} X_{3}
\end{aligned}
$$

Con coeficiente de correlación $R=0,92 ; p<0,001$

Donde: (LNS)

$X_{1}=$ color $($ como opacidad $)$ inicial del licor negro sedimentado

$X_{2}=$ concentración de SST inicial del LNS, $\mathrm{mg} / \mathrm{dm}^{3}$

$X_{3}=$ Tiempo de ozonización

$Y=$ porcentaje de remoción de color $(\% \mathrm{Rc})$

Por ejemplo, para un color inicial del residual líquido bajo estudio de 0,80 ; una concentración de SST inicial de $250 \mathrm{mg} / \mathrm{dm}^{3}$ y, un tiempo de ozonización en el reactor de $120 \mathrm{~min}(2 \mathrm{~h})$; la eficiencia de remoción de color $\left(\% \mathrm{R}_{\mathrm{C}}\right)$, calculada es de $70,48 \%$.

\section{Aspectos económicos}

De acuerdo con los precios actuales de la energía eléctrica, la producción del kilogramo de ozono a partir de aire costaría 0,17 USD, 
aproximadamente; por lo tanto, teniendo en cuenta los resultados obtenidos, la ozonización de licores negros medianamente fuertes procedentes de la industria papelera, que son los predominantes, costaría de 0,26 a 0,28 USD por metro cúbico del mismo, si se sometiera apropiadamente a la sedimentación primaria. Debe señalarse que los costos del proceso disminuyen exponencialmente con la escala del sistema ${ }^{8}$, por lo cual una evaluación económica más precisa requeriría de experiencias a escala piloto, máxime cuando el factor limitante inicial es la difusión del gas.

\section{Conclusion}

d) $\quad$ Los $\% R_{C}$ y $\% R_{D Q O}$ disminuyen al aumentar el color y la $D Q O$ de la muestra a ozonizar en un mismo intervalo de tiempo; esta reducción es más marcada al ser mayor el nivel de SST de la muestra a ozonizar.

e) $\quad \operatorname{Los} \% R_{C}$ y $\% R_{D Q O}$ descienden al incrementarse el nivel de $S S T$ de la muestra a ozonizar.

f) Dependiendo del color inicial, de la $D Q O$ y del nivel de $S S T$ de la muestra, se obtuvieron valores de 10 a $53 \% R_{C}$ al cabo de 10 min y de 66 a $94 \% R_{C}$ al transcurrir $60 \mathrm{~min}$.

\section{References:}

APHA, AWWA-WPCF. 2005. Standard Methods for the Examination of Water and Wastewater. 21th Edition. New York.

CIE 15. 2004. Technical report, colorimetry. Commission Internationale de L'Eclairage.

Chawdury, D.A.; Wikman, B. 2014. Colloidal Stability in Pulp and Paper Industry Process Waters. European Scientific Journal. Edition vol. 10, No. 12.

EPA. 2007. Part III, 40 CFR, Part 122, 136 et al. Guidelines Establishing Test Procedures for the Analysis of Pollutants Under the Clean Water Act: national Primary Drinking Water regulations; and National Secondary Drinking Water Regulations; Analysis and Sampling Procedures; Final Rule. Hunter Lab. 2001. Principios básicos de medida y percepción de color. Información técnica. HUNTER LAB.

Kirk, B. S., McNabney y Wynn, C. S. 1972. Pilot plant studies of tertiary wastewater treatment with ozone. In Ozone in water and wastewater treatment (F. L. Evans, 3th Ed.). Ann Arbor Science Publishers, Inc., Ann Arbor, Michigan, USA.

\footnotetext{
${ }^{8}$ Kirk, B. S., McNabney y Wynn, C. S. (1972) Pilot plant studies of tertiary wastewater treatment with ozone. In Ozone in water and wastewater treatment (F. L. Evans, 3th Ed.). Ann Arbor Science Publishers, Inc., Ann Arbor, Michigan, USA.
} 
Levenspiel, O. 1971. Ingeniería de las Reacciones Químicas. Ed. Reverté, Barcelona, España.

Wark, K; Warner, C.F. 1997. Contaminación del aire. Origen y control. Ed. Limusa. México.

\section{ANEXO}

Figura 1. Estructura aproximada del polímero Lignina

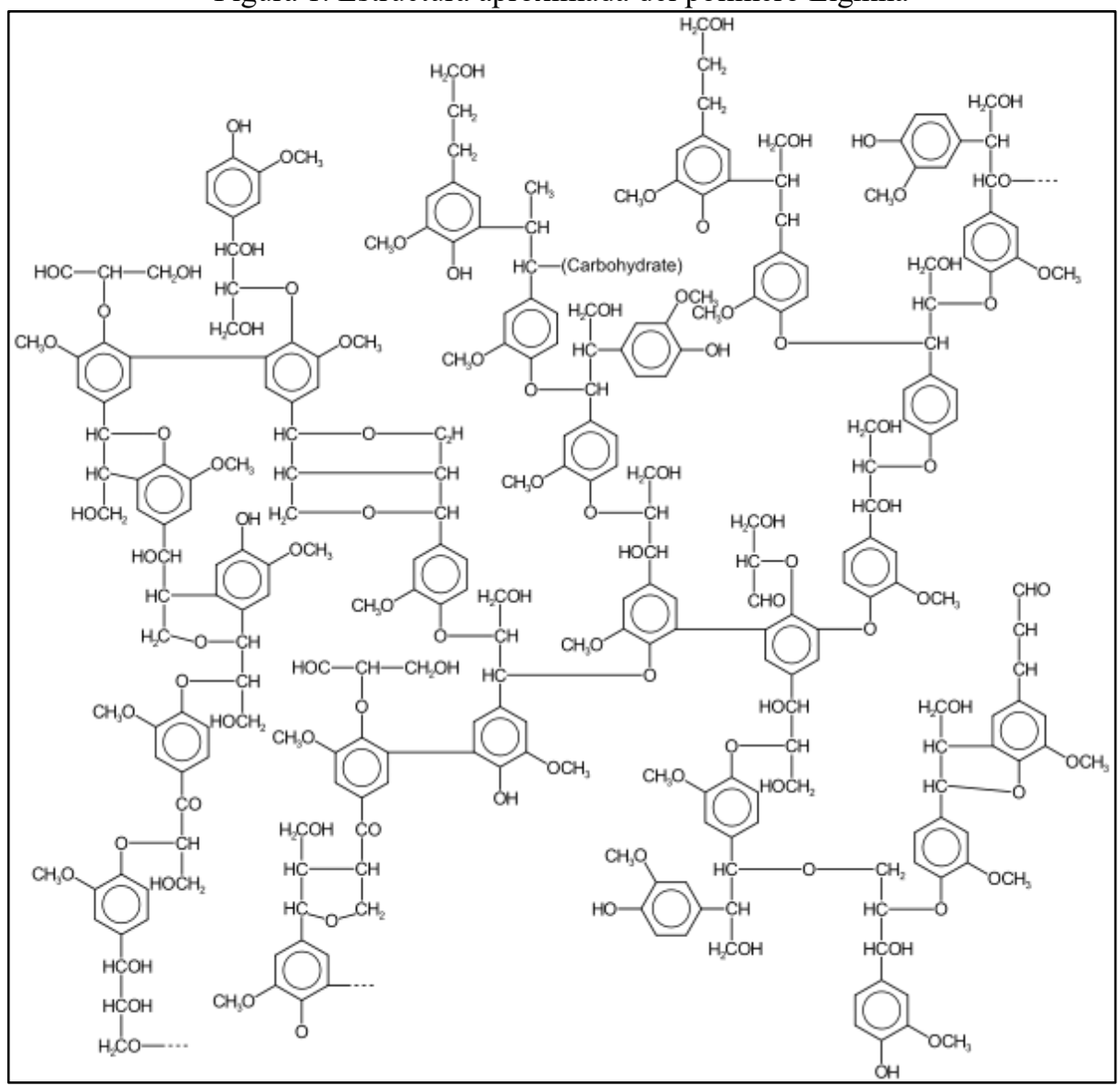

Obtenido de https://es.wikipedia.org/wiki/Lignina el 15 de Noviembre de 2015. 